\title{
Special focus on modeling, analysis and control of stochastic systems*
}

Every aspect in the fields of engineering, natural science, and social science is affected by random phenomena. Dynamic systems, as practical mathematical modeling tools, are also frequently subjected to random influences such as external fluctuations, internal agitation, fluctuating initial conditions, and uncertain parameters.

When building complex dynamic systems, certain lesser-known, poorly understood processes are typically ignored because of limitations in analytical skills and computational capacity. This ignorance always results in uncertainty in dynamic systems. This uncertainty or randomness may have an important influence on the global or local evolution of such systems. Therefore, it is critical to account for randomness in the mathematical modeling of complex dynamic systems. Stochastic dynamic systems are typically appropriate models for such randomly influenced systems and have come to play an important role in many branches of science and industry.

An area of particular interest has been the automatic control of stochastic dynamic systems, with emphasis being placed on the topics of modeling, analysis, and control. Compared with the theory of deterministic dynamic systems, the above-mentioned topics in stochastic dynamic systems are more complex and difficult issues, which have garnered significant attention from researchers. To study these topics in stochastic dynamic systems, one must not only understand the control theory of dynamic systems but also fully understand measurement theory, statistical theory, among others. Therefore, the theory of stochastic dynamic systems is a significant interdisciplinary research field combining control theory, probability theory, and statistics.

Random noise fluctuations are abundant in deterministic dynamic systems. In some cases, noise is not negligible. In other cases, noise may actually be beneficial. It has been demonstrated that noise can not only stabilize a given unstable deterministic dynamic system but also make an already stable deterministic system even more stable.

Furthermore, for a deterministic non-autonomous differential system, which can explode at a finite time, one can introduce polynomial noise to construct a new stochastically perturbed system. It has been demonstrated that polynomial noise can not only guarantee the existence and uniqueness of a global solution for a perturbed system but also make nearly every path in the global solution grow or decay at a particular general rate.

This special focus represents relatively new developments in the modeling, analysis, and control of stochastic systems, where the stochastic maximum principle modeled via Levy process and with Markov switching is investigated through variational methods and filtering techniques. Some results regarding the stabilization of multi-group coupled models via delay feedback control and nonlinear impulsive control are

\footnotetext{
* Citation Deng F Q, Mao X R. Special focus on modeling, analysis and control of stochastic systems. Sci China Inf Sci, 2018, 61(7): 070210, https://doi.org/10.1007/s11432-018-9452-5
} 
reported as application-oriented theories. First, the stabilization of hybrid systems via feedback control based on discrete-time observations is studied. Second, for numerical computations and simulations, the exponential stability of the Euler-Maruyama method for neutral stochastic functional differential equations with jumps is investigated by contrasting it to the continuous model. Third, an interesting topic, the suppression of system explosion via polynomial noise for nonlinear differential systems, is shared. Finally, the sliding mode control for consensus tracking of second-order nonlinear multi-agent systems is introduced. We hope that this special focus will serve as a valuable reference for our readers.

Finally, we would like to express our sincere appreciation to all the authors for submitting their manuscripts. Furthermore, we express our deepest gratitude to all the anonymous reviewers for delivering high-quality and timely review comments. We also thank SCIENCE CHINA Information Sciences Editorial Office for their scrupulous service and support during the entire process of developing this special focus.

Guest Editors:

Feiqi Deng

South China University of Technology, China

Xuerong Mao

University of Strathclyde, UK 